\section{Surface roughness of real operationally used compressor blade and blisk}

\author{
Philipp Gilge' (1), Andreas Kellersmann², Jens Friedrichs ${ }^{2}$ \\ and Jörg R Seume'
}

Proc IMechE Part G:

$\checkmark$ Aerospace Engineering

$0(0) \mathrm{I}-10$

(C) IMechE 2019

\section{(c) (1) (8)}

Article reuse guidelines:

sagepub.com/journals-permissions DOI: 10.1 I 77/0954410019843438 journals.sagepub.com/home/pig

@SAGE

\begin{abstract}
Deterioration of axial compressors is in general a major concern in aircraft engine maintenance. Among other effects, roughness in high-pressure compressor reduces the pressure rise and thus efficiency, thereby increasing the specific fuel consumption of an engine. Therefore, it is important to improve the understanding of roughness on compressor blading and their impact on compressor performance. To investigate the surface roughness of rotor blades of a compressors, different stages of an axial high-pressure compressor and a first-stage blisk (BLade-Integrated-dISK) of a regional aircraft engine is measured by a three-dimensional laser scanning microscope. Fundamental types of roughness structures can be identified: impacts in different sizes, depositions as isotropically distributed single elements with steep flanks and anisotropic roughness structures direct approximately normal to the flow direction. To characterise and quantify the roughness structures in more detail, roughness parameters were determined from the measured surfaces. The quantification showed that the roughness height varies through the compressor depending on the stage, position and the blade side. Overall complex roughness structures of different shape, height and size are detected regardless of the type of the blades.
\end{abstract}

\title{
Keywords
}

Surface roughness, aircraft engine, blisk blades, compressor blades, operationally stressed surfaces

Date received: 14 August 2018; accepted: 15 March 2019

\section{Introduction}

Gas turbine performance deteriorates during flight operation for numerous reasons. A critical area for deterioration which influences significantly the aerodynamic performance is the blade surface. In this paper, the roughness of first-stage rotor blades of high-pressure compressors is part of the investigation. The task of compressors is to increase the total pressure. For this reason, compressor airfoils are at great risk for boundary layer separation which decreases stage efficiency and may lead to stall or surge. The risk of these phenomena depends mainly on Reynolds and Mach numbers and of course roughness. $^{1}$

Leipold et al. $^{2}$ explored the critical role of the Reynolds number in a high-pressure cascade in conjunction with surface roughness. They varied the Reynolds number from $3 \times 10^{5}$ to $1 \times 10^{6}$ based on inlet conditions. The inlet Mach Number was set to 0.67 to generate a peak Mach Number close to $\mathrm{Ma}=1$. An important finding was that the loss coefficient rises with the Reynolds Number. Above $5 \times 10^{5}$ thin boundary layers lead to turbulent boundary layer separation on the suction side of rough blades.
Particularly for high-pressure compressors with Reynolds Numbers of $1 \times 10^{6}$ and higher, this effect is relevant. Back et al. ${ }^{3}$ found equivalent results in a low-speed compressor cascade. Like Leipold et al., ${ }^{2}$ they found decreasing performance at a critical Reynolds Number of about $6 \times 10^{5}$ with a critical roughness $R a$ between 2.0 and $2.9 \mu \mathrm{m}$. The cascade performance is mainly reduced by roughness on the suction side but an entirely rough blade reduces the performance even further. The main focus of their studies was on the mass-averaged profile losses, which is mostly affected by the suction side in the downstream half-chord of the blade. Another important result of their study was that an increase of the area covered with roughness decreases the critical

\footnotetext{
'Institute of Turbomachinery and Fluid Dynamics, Leibniz University Hannover, Hannover, Germany

${ }^{2}$ Institute of Jet Propulsion and Turbomachinery, TU Braunschweig, Braunschweig, Germany
}

\section{Corresponding author:}

Philipp Gilge, Institute of Turbomachinery and Fluid Dynamics, Appelstr. 9. Hannover 30167, Germany.

Email: gilge@tfd.uni-hannover.de 
Reynolds number. Schaffler ${ }^{4}$ found the same trend in a five-stage high-pressure compressor rig. He reported lower polytrophic efficiency beginning for a chordbased Reynolds Number of $3.1 \times 10^{5}$ in the first rotor. He analysed the machining roughness of different manufacturing techniques of blades, leading to different roughness results.

Bammert et al. ${ }^{5}$ investigated a low-speed compressor cascade with five different roughness levels at a fixed Reynolds Number $4.3 \times 10^{5}$ and an Inlet Mach Number of 0.11 . They used sand grain roughness levels $k_{s} / \mathrm{c}$ from $2.3 \times 10^{-4}$ to $5.6 \times 10^{-3}$. They determined increasing profile losses by raising the sand grain roughness. With higher roughness at the trailing edge, separation occurs on the suction side along with a reduced turning. Because of the effect of the reduced turning on the following blade row, Bammert et al. ${ }^{5}$ investigated comparable roughness levels in a three-stage axial compressor. All airfoils were roughened equally. The complete rig suffered from $6 \%$ to $13 \%$ loss of efficiency and an overall pressure ratio reduction by $30 \%$ with respect to their comparative configuration.

Suder et al. ${ }^{6}$ analysed a transonic compressor rotor while adding roughness and thickness to the blades. The roughness was applied by coating the blades, which increased the leading edge thickness by $10 \%$ at the hub and $20 \%$ at the tip. The coated configuration had a roughness $R q$ between $0.5 \mu \mathrm{m}$ and $3.2 \mu \mathrm{m}$. Because of this procedure, the roughness could be studied without mixing the effects of adding roughness and increasing blade thickness. The highest rotor roughness results in a $9 \%$ pressure ratio loss with regard to the baseline rotor. Suder et al. ${ }^{6}$ also investigated the influence of roughness position along the blade. They can show that the first $2 \%$ of distance from leading edge affects the boundary layer thickness and therefore the interaction with the shock. This results in an increased blockage and a reduced diffusion in the blade passage. Gbadebo et al. ${ }^{7}$ examined the influence of surface roughness on stator blades of a single-stage low-speed axial compressor. They used a centre-line averaged roughness of about 1.5 to $2.0 \mu \mathrm{m}$ obtained from a turbofan engine after a long period of airline operation. The roughness was applied by a strip covering $50 \%$ to $100 \%$ of span and about $20 \%$ of chord length at different positions. Their experiments showed that roughness induces a larger hub-corner-separation which resulted in high losses, increased blockade and deviation compared to their baseline experiment. The three-dimensional end wall separation was triggered by a leading edge roughness extending the affected area up to $30 \%$ of the span compared to the baseline configuration.

The impact of surface roughness on axial compressor performance was studied by Syverud and Bakken. ${ }^{8}$ They compared performance test data of the GE J85-13 engine with loss models addressing friction, blockage and deviation. They used the same approach for surface roughness characterisation like Gbadebo et al. ${ }^{7}$ Their results show a gap between the model prediction and measured test data of a real engine. However, similar to Suder et al., ${ }^{6}$ the results give a hint how important surface roughness due to compressor performance can be.

In addition, the roughness effect due to erosion and fouling is another effect which has to be considered. Fouling describes the process of deposition of particles on the surface of blades due to operational pollution. Another critical effect is a change of roughness and geometry due to deposits. Syverud and Bakken ${ }^{9}$ showed that deposits of different types such as salt or oil have the same impact on the performance of a compressor. As done by Gbadebo et al. ${ }^{7}$ and Syverud and Bakken, ${ }^{8,9}$ the roughness in most of the literature is measured only at few discrete points on the blade but applied on the whole blade with the assumption of conformity. However, it was shown that local variations in the roughness on a compressor blade can lead to different loss increases depending on the roughness position. ${ }^{10,11}$ As a result, a detailed analysis of the roughness distribution over the blade surface and the structures itself is important to understand and model the effects of real surface roughness in engines.

\section{Roughness measurements and quantification}

All investigated roughnesses were measured with confocal laser scanning microscopes of the same type (Keyence VK-X200). The measurements are contact free, and a three-dimensional representation is obtained. The used laser scanning microscope has two optical paths. One for a white-light source with a CCD chip for high-resolution colour pictures and a second optical path using a laser diode with a wavelength of $658 \mathrm{~nm}$. To obtain highly detailed pictures of the surface-roughness, an objective lens with $20 \times$ magnification was used for the single-blade measurements, and for the blisk blade, a lens with $50 \times$ magnification was used. The size of each measurement patch with a $20 \times$ magnification is $704 \mu \mathrm{m}$ by $528 \mu \mathrm{m}$ and $270 \mu \mathrm{m}$ by $203 \mu \mathrm{m}$ for a magnification of $50 \times$. For both cases, the physical resolution is 1024 by 768 pixels per pitch. In addition, the measurements of the $20 \times$ magnification are stitched together to receive a wider measurement field. In total, four measurements are stitched together with a final resolution of 1296 by 1863 pixels and an area size of $890 \mu \mathrm{m}$ by $1280 \mu \mathrm{m}$. To resolve the picture in the height axis, the surface scan is replicated from the bottom of the sample to the scanned surface.

Measurement error of the used Keyence microscope is given by the manufacture and depends on the vertical measurement range $\left(\Delta h_{M R}\right)$

$$
h_{\text {err }}=0.2+\Delta h_{M R} / 100
$$


For a good comparability, the measurement range $\left(\Delta h_{M R}\right)$ is set in dependency of the magnification factor. In this investigation, a measurement error for $20 \times$ magnification of $h_{\text {err } 20 x}= \pm 0.93 \mu \mathrm{m}$ and $50 \times$ magnification of $h_{\text {err }} 50 x= \pm 0.58 \mu \mathrm{m}$ is determined. To verify the given measurement errors, a test surface is measured 30 times for both magnification factors. The test surface is created by a smooth surface with silicon grains to realise a roughness including steep flanks. Steep flanks are difficult to measure using a vertical optical measurement system and entail a higher level of measurement errors. The mean standard deviation $\overline{h_{i, s t d}}$ is calculated from the 30 repetition measurements resulting in $\overline{h_{i, s t d} 20 x}= \pm 0.96 \mu \mathrm{m}$ and $\overline{h_{i, s t d} 50 x}= \pm 0.46 \mu \mathrm{m}$ which is in good agreement with the given errors of the microscope manufacturer. In addition, the confidence interval $\overline{h_{i, v a r}}$ based on a confidence level of $99 \%$ is estimated. The results of $\overline{h_{i, \text { var } 20 x}}= \pm 0.48 \mu \mathrm{m}$ and $\overline{h_{i, \text { var } 50 x}}= \pm 0.23 \mu \mathrm{m}$ show that the standard deviation is dominated by single outliers resulting from the steep flank measurements. Thus, the real measurement error for the presented study is smaller than the official error estimation of the microscope manufacturer. To give a measurement and determination error for the following investigation, the relative error is given by the estimated confidence intervals divided by the mean roughness height of the test surface (representing the mean roughness height $R a$ explained in the following paragraph) for the both magnification factors. All results of the $20 \times$ magnification have an error of $\pm 1.1 \%$ and those of the $50 \times$ magnification an error of $\pm 1.4 \%$. For a DIN-standard measurement, a cut-off length has to be specified depending on the anticipated surface roughness. Based on Gbadebo et al., ${ }^{7}$ the anticipated roughness $R a$ is between $0.1 \mu \mathrm{m}$ and $2.0 \mu \mathrm{m}$ which leads to a cut-off length $\lambda_{c}$ of $0.8 \mathrm{~mm}$. For a standard measurement, this cut-off length requires a measurement length of $4.8 \mathrm{~mm}$. Because of a strong curvature and measurement limits, this requirement cannot be fulfilled in the present application. For a quantitative characterisation of different surface roughnesses, a range of statistical parameters is calculated from the surface measurements. In the present work, values of the mean roughness height $R a$, the average root-mean-squared roughness $R q$ and roughness peak-to-valley height $R z$ are used to parametrise the surface roughness. These values were defined by Thomas $^{12}$ for a discrete height profile as:

$$
\begin{aligned}
& R a=\frac{1}{N} \sum_{i=1}^{N}\left|h_{i}\right| \\
& R q=\sqrt{\frac{1}{N} \sum_{i=1}^{N} h_{i}^{2}} \\
& R z=h_{\text {max }}-h_{\text {min }}
\end{aligned}
$$

The above-mentioned parameters are defined for two-dimensional surface measurements. Threedimensional surface data are divided into several tracks with a 1 pixel width. Roughness parameters were calculated for each track individually and all tracks are directed in the flow direction. Finally, the average value of the determined parameters was evaluated. This method guarantees that the complete measured surface is taken into account for the determination of the statistical parameters. Considering the position of the blade, all measurements performed in this work were equally aligned.

To characterise a surface from an aerodynamic point of view, the shape and density parameter $\Lambda_{S}$ was defined by Sigal ${ }^{13}$ and modified by Bons ${ }^{14}$ for two-dimensional roughness functions $\mathrm{h}(\mathrm{x})$

$$
\Lambda_{s}=\frac{\sum d x_{i}}{\sum \Delta h_{i}}\left(\frac{\sum \Delta h_{i}}{\sum l_{i}}\right)^{-1.6}
$$

with $d x_{i}$ the streamwise distance, $\Delta h_{i}$ the step height and $l_{i}$ the local surface wetted distance given by $l_{i}=\sqrt{\Delta h_{i}^{2}+d x_{i}^{2}}$. In order to calculate $k_{s}$, the correlation

$$
\frac{k_{s}}{k}=0.43 \cdot \log \left(\Lambda_{S}\right)+0.82
$$

of Bons ${ }^{14}$ with a roughness height $k$ is used. In this study, the roughness height $k$ is equal to the mean roughness height $R a{ }^{15}$ All Parameters are calculated in the flow direction represented in this study by the $\mathrm{x}$-direction.

One challenge for the measurements on the blisk is the small passages between two rotor blades. The pitch between two blades is too small to measure the surface roughness directly. Another challenge for the singleblade roughness was measuring the roughness directly from individual high-pressure compressor blades which were on ground only for service. To use the microscope for direct measurements on the single blades would take too much time. Also, to avoid distortions because of the handling of the blades after the complete removal from the compressor module, the surface structures must be preserved as early as possible. To solve both problems, an indirect measurement method was used by applying a casting compound based on a two-component silicone to take impressions. Due to this measurement strategy, an indirect measurement of the surface structures with the laser scanning microscope could be performed.

In order to verify the method, direct test measurements of a blade roughness were compared to indirect measurements using a casting compound. Figure 1 shows measurements of a real surface and of a casting compound of the same surface. The casting compound measurement is presented inverted. The same structures can be seen in both figures in a proper resolution to analyse the surface structures. The average 


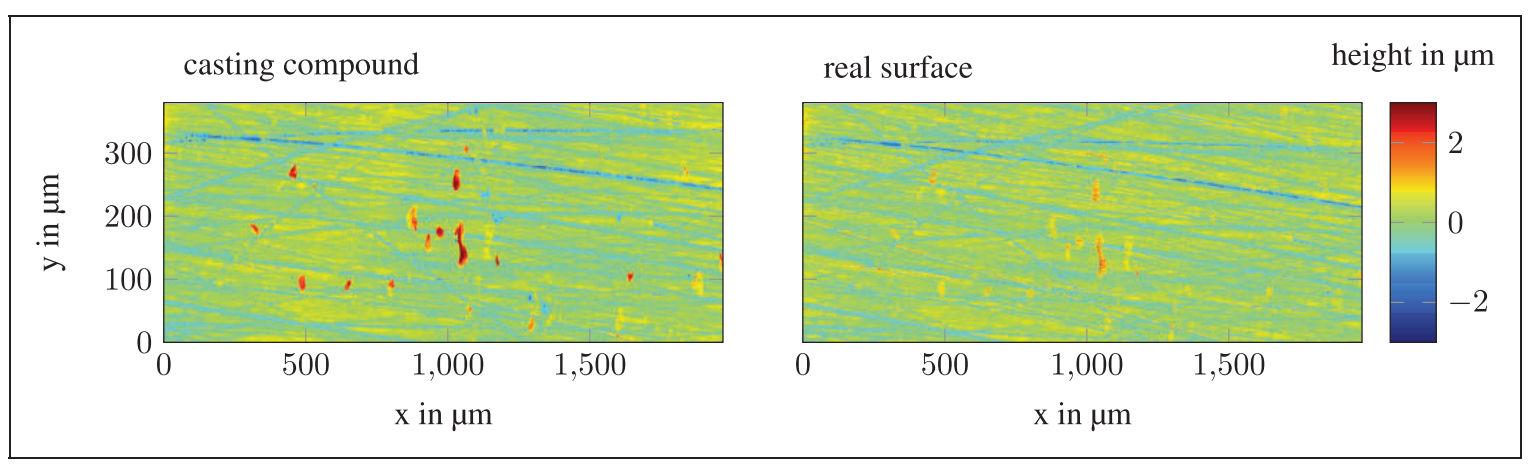

Figure I. Qualitative verification of indirect roughness measurements with casting compound and the direct measurement of a reference surface roughness.

Table I. Measured surface roughness from the test surface for the direct measurement $A$ and indirectly with casting compound measurement $B$.

\begin{tabular}{lll}
\hline & Av. Ra in $\mu \mathrm{m}$ & Av. Rz in $\mu \mathrm{m}$ \\
\hline A & 0.257 & 4.021 \\
B & 0.244 & 4.439 \\
\hline
\end{tabular}

roughness $R a$ was determined for both measurements and differs by about $0.013 \mu \mathrm{m}$. The average surface roughness $R z$ differs by about $0.418 \mu \mathrm{m}$ (Table 1 ).

\section{Measured surfaces}

The roughness measurements were performed on real operationally used blades from high-pressure compressors. The surfaces of two different types of aircraft engines and blades were measured (summary in Table 2).

Single-blade roughness was measured in highpressure compressors of a medium size high-bypass aircraft engine in an under-wing position. The engine has been in operation in flight regions with little natural environmental or anthropogenic effects. ${ }^{16}$ The blades are measured after a typical flight operation time of 20,000 cycles by the Institute of Turbomachinery and Fluid Dynamics at the Leibniz University Hannover. The measured blisk blades roughness was measured from a blisk, installed in a rear-mounted engine of a regional aircraft with short flight cycles, which has been in operation for about 20,000 cycles, too. The roughness measurements on the blisk were performed by the Institute of Jet Propulsion and Turbomachinery at the Technical University of Braunschweig. Both measurements were performed by using the same type and model of microscope.

The roughness of the single-blade high-pressure compressor blades was measured at four positions on the suction side and four positions on the pressure side at mid-span. The first position is at the leading edge, the second position at $30 \%$ of the chord length,
Table 2. Summary of the origin of the measured surfaces.

Single blade

Blisk blade
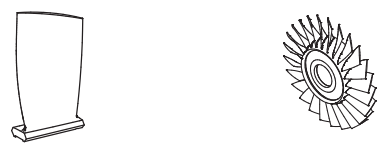

\begin{tabular}{|c|c|c|}
\hline Engine type & Two-spool & Two-spool \\
\hline Engine size & Medium-size & Small \\
\hline Engine position & Under-wing & Rear-mounted \\
\hline Operation cycles & 20,000 & 20,000 \\
\hline Engine module & $\begin{array}{l}\text { High-pressure } \\
\text { compressor }\end{array}$ & $\begin{array}{l}\text { High-pressure } \\
\text { compressor }\end{array}$ \\
\hline Measured stages & First, centre, last & First \\
\hline Blade side & $\begin{array}{l}\text { Pressure side, } \\
\text { suction side }\end{array}$ & $\begin{array}{l}\text { Pressure side, } \\
\text { suction side }\end{array}$ \\
\hline Measured position & $\begin{array}{c}\text { Four positions } \\
\text { at mid-span }\end{array}$ & Whole blade \\
\hline Number of blades & 25 per stage & II \\
\hline
\end{tabular}

the third position at $60 \%$ of the chord length, and the fourth position near the trailing edge at $90 \%$ of the chord length. To get an impression of the roughness distribution through a high-pressure compressor, three stages are investigated: The first-stage blades at the inlet, the centre stage blades, and the last stage blades. From each stage, the roughness of five blades were measured. Overall blades from five engines were investigated ( 25 blades per stage).

The blisk blade roughness is measured over the whole surface of the pressure side and the suction side. The measurement grid is shown in Figure 4. In total, 14 blades of one blisk were measured.

\section{Results}

\section{Single-blade roughness}

The results of the roughness quantification of the single-blade roughness are summarised separately 




Figure 2. Results of the roughness measurements on the single-blade roughness from a high-pressure compressor of a mid-size aircraft engine. The error bars represent the standard derivation of the performed measurements on the specific position and not the measurement error of the measurement system.

for the pressure side and the suction side in Figure 2. The mean values for each stage are calculated with the corresponding standard deviation. The standard deviation contains the systematic measurement error and the variation of the roughness measurements. Thus, the standard deviation can be seen as a measure of the variation of the roughness height itself in each measurement position. A position with a high standard deviation shows higher level of variations in the roughness than positions with a lower standard deviation.

The pressure side roughness quantification shows entirely a uniform distributed roughness height $10 \mu \mathrm{m}$ along the chords of stages, excepting the first stage. In the first stage, a higher level of the roughness height is 
detectable. The values for the equivalent sand grain roughness $k_{s}$ vary from $24 \mu \mathrm{m}$ at the leading edge to $10 \mu \mathrm{m}$ in the trailing edge region. The roughness distribution for the centre stage and the last stages shows only slight variations along the chord. Especially, the sand grain roughness height for the roughness of the centre stage is nearly constant and varies around $10 \mu \mathrm{m}$. The roughness height of the last stage is overall the lowest but follows the trend of the first-stage roughness height by having its maximum at the leading and lower roughness height in the centre and near the trailing edge. The standard deviation of the leading edge roughness of the first stage is $\pm 5 \mu \mathrm{m}$ and for the other stages and positions on the pressure side approximately $\pm 3 \mu \mathrm{m}$.

To correlate the determined roughness parameters with the real surface roughness structures, a qualitative analysis of the roughness structures is performed. Examples for representative roughness structures for each stage and position on the blade are shown in Figure 3. On the pressure side, different fundamental structures in the roughness are observed. A mix of impacts characterised by round depressions of different size and erosion or depositions characterised by isotropic distributed roughness elements of different size are detected. Correlated to the highest values of roughness height on the leading edge of the first stage, the biggest roughness elements can be detected in the qualitative analysis, too. This roughness mainly consists of isotropic roughness elements which can also be detected in smaller size, at the $x / c=0.3$ position of the first stage and in the centre stage blades. Because of the high level of roughness, a clear detection of impacts or deposition is not possible

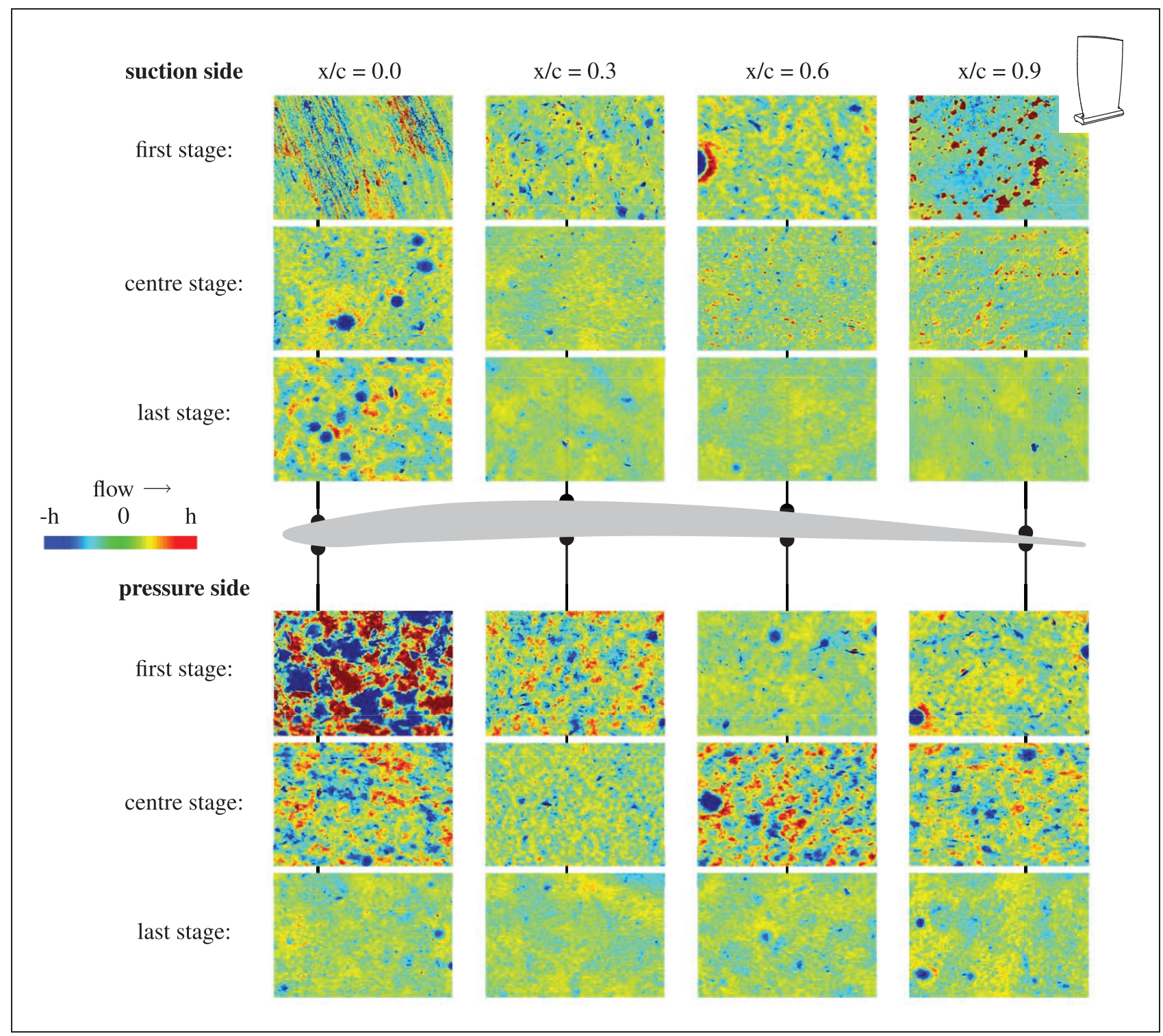

Figure 3. Examples of single-blade roughnesses from a high-pressure compressor of a mid-size aircraft engine from different stages and measurement positions. The size of the measurement area is $0.890 \mathrm{~mm}$ by $1.280 \mathrm{~mm}$. 


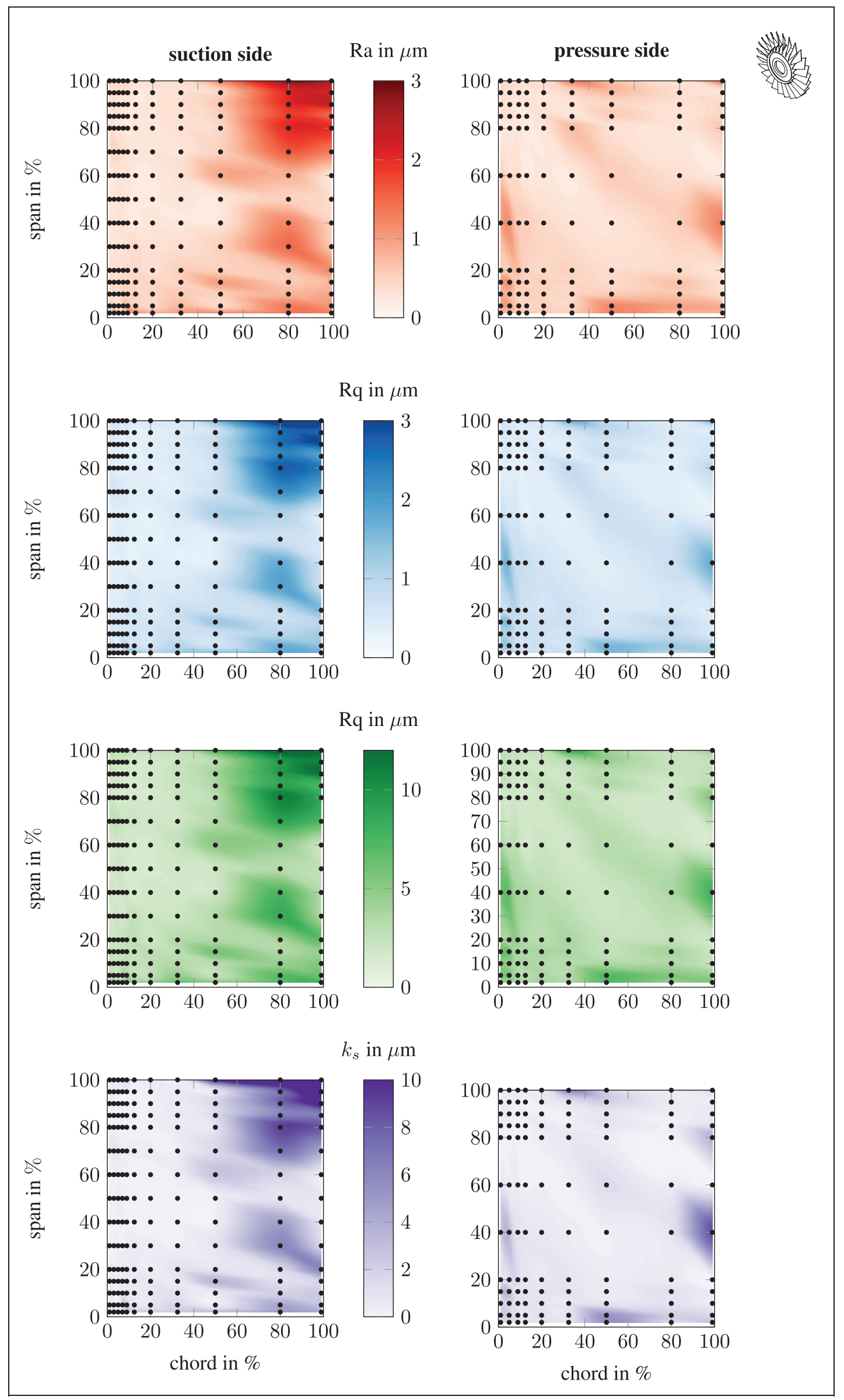

Figure 4. Results of the roughness quantification of the blisk blade roughness measurements. The black dots are the measurement positions. The colour plot is a cubic interpolation to show the roughness distribution over the whole surface. 
in this investigation. In other investigations, depositions are found in this region, ${ }^{17}$ as well as a high density of impacts because of particles. ${ }^{18}$ From these observations, the high degree of roughness seems to be the result of the high particle loaded flow at the inlet of the compressor. In all stages of the pressure side, impacts of different size are observed which is in good agreement with observations of Tabakoff. ${ }^{18}$

The roughness height distribution on the suction differs partly from the pressure side roughness. Especially, no increased roughness height at the leading edge of the first stage is detected, if only the standard parameters $(R a, R q$ and $R z)$ are considered. However, an increased roughness height is detected by using the $k_{s}$ parametrisation. A roughness height of $k_{s}=22 \mu \mathrm{m}$ is determined with a high standard deviation of $\pm 15 \mu \mathrm{m}$. The high standard deviation indicates high fluctuations in the roughness size and shape. An explanation can be given by looking at the roughness structures itself on this position (Figure 3). Only on this position mainly, anisotropic structure can be detected. The structures are directed in a normal direction to the flow. The dependency of the $k_{s}$ determination method leads to a higher sensitivity of this parameter for the anisotropy and direction of the roughness structures. Other investigations showed ${ }^{19,20}$ that because of oil leakage from the bearing system of the engine, sticky liquid can enter the flow path and is transported along the blades because of the centrifugal force. At the leading edge region, particles will only stick on the surface with this oil, because otherwise particles will be transported further downstream, because of the high shear stresses near the wall. ${ }^{19}$ With lower shear stress rates further downstream on the blades, more and more deposition can build up resulting in higher values for the roughness height in all roughness parameters. Especially, in the first stage, a significant increase can be detected in the trailing edge region. The roughness structure shape is isotropically scattered with steep flanks and represents depositions. ${ }^{17,19,20}$ The size and quantity of the depositions decrease through the compressor, and at the trailing edge region of the last stage almost no depositions are detectable.

\section{Blisk blade roughness}

The mean values of the measured blisk roughnesses are summarised in Figure 4 for all four determined roughness parameters $\left(R a, R q, R z\right.$ and $\left.k_{s}\right)$ and all measured positions of the blisk blade. The colour map is created by a cubic interpolation of the mean values of the measurements to visualise the changes in the roughness over the blade.

The overall mean of the measured roughness height on the suction side is $R a=0.60 \mu \mathrm{m}(R q=0.78 \mu \mathrm{m}$, $\left.R z=3.35 \mu \mathrm{m}, k_{s}=1.60 \mu \mathrm{m}\right)$, but it varies along the chord, in particular in the tip region. In the tip region, the highest roughness heights are detectable ( $R a=3.04 \mu \mathrm{m}, \quad R q=3.74 \mu \mathrm{m}, \quad R z=14.35 \mu \mathrm{m}, \quad k_{s}=$ $17.05 \mu \mathrm{m})$ near the trailing edge. In positions on the suction side, the roughness structures consist of two fundamental shapes, impacts and isotropic distributed roughness elements of different size (Figure 5). No anisotropic structures are detectable on the measured surfaces. The roughness height distribution correlates with observation of other investigations. The lower shear stress on the surface and separation, e.g. because of the tip vortex in the tip region on the suction side, leads to good conditions for depositions. The depositions cause big roughness structures. ${ }^{19-22}$

On the pressure side, an overall mean of $R a=0.6 \mu \mathrm{m} \quad\left(R q=0.76 \mu \mathrm{m}, R z=3.47 \mu \mathrm{m}, \quad k_{s}=\right.$ $1.17 \mu \mathrm{m})$ is determined. Also, slight variations along the chord and the span are detectable, but these variations are smaller compared to the suction side roughness. The higher values are located near the edges of the blade and have maxima of $R a=1.85 \mu \mathrm{m}(R q=$ $\left.2.14 \mu \mathrm{m}, \quad R z=9.23 \mu \mathrm{m}, \quad k_{s}=8.78 \mu \mathrm{m}\right)$. The overall roughness height of the blisk blade roughness is equal to the single-blade roughness. In particular, the roughness distribution along the chord on the suction side shows a similar trend, with higher roughness values in the trailing edge region on the blisk. This may result from the different sizes of the investigated engines. Smaller engines such as the blisk-bladed engine of the presented investigation lead to more fouling. ${ }^{22}$ However, the roughness on the blisk blade near the mid-span is equally distributed, and the blisk

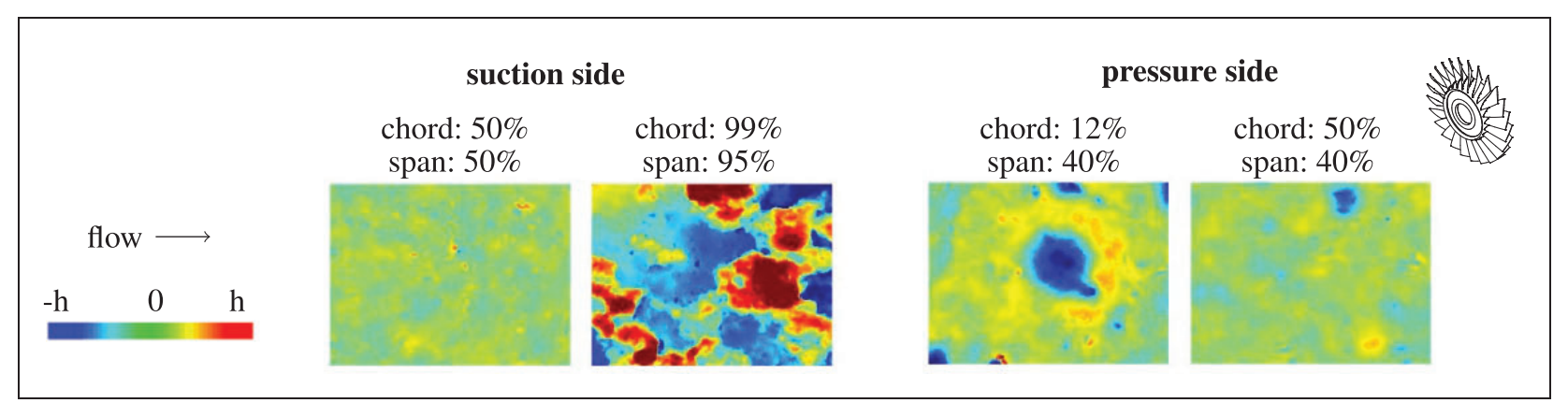

Figure 5. Examples of the blisk blade roughness of a small aircraft engine. The size of the measured area is $0.270 \mathrm{~mm}$ by $0.203 \mathrm{~mm}$. 
blades and all of the roughness parameters in this region show the same trend.

\section{Conclusions and outlook}

The investigated real surface roughness of operationally used compressor blades of an under-wing mid-size aircraft engine and blisk blades of a small-size rearmounted shows complex roughness structures depending on stage, position and side of the blade, and also the type of the engine. The variations are detectable in the roughness height (quantified by roughness parameters $R a, R q, R z$ and $k_{s}$ ) and also qualitatively in the shape of the roughness structures. The biggest roughness height is detected in the first stage followed by the centre stage and the last stage of the compressor. Fundamental types of roughness structures can be identified: impacts as round depressions in different sizes, depositions as isotropically distributed single elements with steep flanks in different size and anisotropic roughness structures. However, anisotropic structures are detectable only at the leading edge of the single-blades in the first stage of the mid-size engine. These structures are oriented at an angle ranging from $45^{\circ}$ to $90^{\circ}$ normal to the flow direction and are the result of leakage oil and flow particles. These structures lead to a higher equivalent sand grain roughness $\left(k_{s}\right)$ magnitude, which is not detectable in the standard roughness parameters $(R a, R q$ and $R z)$. On the pressure side, the highest roughness is detected at the leading edge, identified as a mix of erosion, impacts and depositions. Further downstream, an increased density of depositions is detected, resulting in increased roughness values near the trailing edge on the suction side. This effect decreases with increasing number of the stage as well as the roughness height. On the blisk blade, the biggest roughness height and structures are detected in the tip region on the suction side, resulting from lower shear stress rates and the tip vortex, and correlate with the observations of other investigations.

After characterisation and quantification of the compressor blade roughness, further investigation will be performed to determine the effect of the measured complex roughness on the compressor blade performance. Therefore, the near wall flow and the blade losses because of the roughness will be investigated in detail to develop or enhance surface roughness models and to improve the prediction of the roughness effect on the flow. It is also planned to publish the surface data used for presented work. ${ }^{23}$

\section{Declaration of Conflicting Interests}

The author(s) declared no potential conflicts of interest with respect to the research, authorship, and/or publication of this article.

\section{Funding}

The author(s) disclosed receipt of the following financial support for the research, authorship, and/or publication of this article: The present work has been carried out in the subprojects B3 and D4 within the Collaborative Research Center (CRC) 871 'Regeneration of Complex Capital Goods', which is funded by the DFG (Deutsche Forschungsgemeinschaft) under grant of CRC871. The authors would like to thank the DFG for their support.

\section{ORCID iD}

Philipp Gilge (D) http://orcid.org/0000-0002-5719-3267

\section{References}

1. Bons JP. A review of surface roughness effects in gas turbines. $J$ Turbomach 2010; 132: 021004.

2. Leipold R, Boese M and Fottner L. The influence of technical surface roughness caused by precision forging on the flow around a highly loaded compressor cascade. $J$ Turbomach 2000; 122: 416-424.

3. Back SC, Hobson GV, Song SJ, et al. Effects of Reynolds number and surface roughness magnitude and location on compressor cascade performance. J Turbomach 2012; 134: 051013.

4. Schaffler A. Experimental and analytical investigation of the effects of Reynolds number and blade surface roughness on multistage axial flow compressors. J Eng Gas Turbines Power 1980; 102: 5-12.

5. Bammert $\mathrm{K}$ and Milsch R. Boundary layers on rough compressor blades. In: ASME 1972 international gas turbine and fluids engineering conference and products show, p. V001T01A047. New York, NY: American Society of Mechanical Engineers, 1972.

6. Suder KL, Chima RV, Strazisar AJ, et al. The effect of adding roughness and thickness to a transonic axial compressor rotor. In: ASME 1994 international gas turbine and aeroengine congress and exposition, p. V001T01A113. New York, NY: American Society of Mechanical Engineers, 1994.

7. Gbadebo SA, Hynes TP and Cumpsty NA. Influence of surface roughness on three-dimensional separation in axial compressors. In: ASME turbo expo 2004: power for land, sea, and air, pp.471-481. New York, NY: American Society of Mechanical Engineers, 2004.

8. Syverud E and Bakken LE. The impact of surface roughness on axial compressor performance deterioration. In: ASME turbo expo 2006: power for land, sea, and air, pp.491-501. New York, NY: American Society of Mechanical Engineers, 2006.

9. Syverud E and Bakken LE. Axial Compressor Deterioration Caused by Saltwater Ingestion. J Turbomach 2007; 129: 119-126.

10. Gilge P, Seume JR and Mulleners K. Analysis of local roughness combinations on the aerodynamic properties of a compressor blade. In: AIAA aerospace sciences meeting. Reston, VA: American Institute of Aeronautics and Astronautics, 2018.

11. Gilge P, Seume JR and Mulleners K. Effect of surface roughness on the characteristic length scales in the wake of a compressor blade: a pod analysis. In: Proceedings of the 19th international symposium on application of laser and imaging techniques to fluid mechanics, Lisbon, Portugal, 16-19 July 2018.

12. Thomas TR. Characterization of surface roughness. Precis Eng 1981; 3: 97-104. 
13. Sigal A and Danberg JE. New correlation of roughness density effect on the turbulent boundary layer. AIAA J 1990; 28: 554-556.

14. Bons JP. A critical assessment of Reynolds analogy for turbine flows. ASME J Heat Transfer 2005; 127 : 472-485.

15. Hohenstein $\mathbf{S}$ and Seume J. Numerical investigation on the influence of anisotropic surface roughness on the skin friction. In: Proceedings of the European turbomachinery conference, Lappeenranta, Finland, 15-19 April 2013.

16. Ebmeyer C, Friedrichs J, Wensky T, et al. Evaluation of total engine performance degradation based on modular efficiencies. In: ASME 2011 turbo expo: turbine technical conference and exposition, pp.201-210. New York: American Society of Mechanical Engineers, 2011.

17. Suman A, Morini M, Kurz R, et al. Estimation of the particle deposition on a transonic axial compressor blade. J Eng Gas Turbines Power 2015; 138: 012604.

18. Tabakoff W. Compressor erosion and performance deterioration. J Fluids Eng 1987; 109: 297.
19. Kurz R, Musgrove G and Brun K. Experimental evaluation of compressor blade fouling. J Eng Gas Turbines Power 2016; 139: 032601.

20. Kurz R and Brun K. Fouling mechanisms in axial compressors. J Eng Gas Turbines Power 2012; 134: 032401.

21. Suman A, Morini M, Kurz R, et al. Quantitative computational fluid dynamic analyses of particle deposition on a transonic axial compressor blade-part II: impact kinematics and particle sticking analysis. $J$ Turbomach 2014; 137: 021010.

22. Tarabrin AP, Schurovsky VA, Bodrov AI, et al. An analysis of axial compressor fouling and a blade cleaning method. J Turbomach 1998; 120: 256.

23. Gilge P, Kellersmann A, Kurth S, et al. Dataset: Surface roughness of real operationally used compressor blade and blisk 2019. Research Data Repository of the Leibniz University of Hannover. Available at: https:// doi.org/10.25835/0084372. 